\title{
Characterization of Ferroelectric Random Access Memory (FRAM) Storage Capacitors
}

\author{
Sahar Hihath, Hannah Harter, Jerry Fortier and David Flowers \\ Defense Microelectronics Activity (DMEA), McClellan, California, United States
}

Ferroelectric materials have unique properties including polarization hysteresis, high permittivity, and enhanced piezoelectric effects. These properties make ferroelectric materials good candidates for applications such as non-volatile memories, capacitors, and sensors. [1] Specifically, ferroelectric random access memory (FRAM) has gained attention over the past decade due to low power consumption and high read/write endurance when compared to flash non-volatile memory technologies. Most ferroelectric materials remain in a polarized state over a limited temperature range. As temperature increases, depolarization gradually occurs until a fully depolarized state is reached at the Curie temperature. Therefore, it is important to characterize the material structure and chemical state/composition of the FRAM data storage capacitors as their properties impact the long-term reliability of FRAM products. The most commonly used materials used in FRAM storage capacitors are lead-zirconium-titanate or $\mathrm{Pb}[\mathrm{Zr} 1$ xTix]O3 (PZT) and lead-free materials such as BaTiO3. [2] In this report, we investigate the reliability of FRAM storage capacitors embedded in a microcontroller using the Scanning Electron Microscope (SEM) imaging and Energy Dispersive X-ray Spectroscopy (EDXS) to determine the structure and the elemental composition of the ceramic storage capacitor.

To characterize the device, commercial off the shelf package parts were purchased and de-capsulated to extract the Integrated Circuit (IC) die via chemical etching with $100 \mathrm{~mL}$ sulfuric acid at $60^{\circ} \mathrm{C}$ for 8 hours. [3] To mitigate charging while imaging with an electron microscope, the surface of the die was coated with a thin layer of palladium as the die surface is typically covered with a passivation layer of Si3N4. Scanning electron microscope (SEM) imaging was performed with the FEI Helios 660 operated at $1 \mathrm{kV}$ in secondary electron (SE) mode with a Through the Lens Detector (TLD) detector. Scanning transmission electron microscope in scanning electron microscopy (STEM-in-SEM) method was performed with the FEI Helios 660 operated at $23 \mathrm{kV}$ in secondary electron (SE) mode with an STEM 3+ detector. EDXS analysis was done with the FEI Helios 660 operated at $20 \mathrm{kV}$.

To understand the structure and composition of the FRAM capacitor structures, SEM imaging and EDXS analysis were sequentially conducted. Figure 1A shows an array of FRAM capacitors, located between the metal one layer and the contacts to the substrate fabrication layers. To gain more insight into the structure and the composition of the FRAM modules, STEM-in-SEM method was utilized to capture highangle annular dark-field (HAADF) images. Figure 1B illustrates an HAADF image with labeled regions, important for compositional analysis. The labeled regions in Figure 1B are tentatively identified as TiAlN (U), Ir (V), IrO2 (W), PZT (X), Ir (Y), and TiAlN (Z). [4] Figure 2A is an SEM image utilized as a survey during EDXS acquisition. The EDXS profile for $\mathrm{Ti}, \mathrm{Zr}$, and $\mathrm{Pb}$ is shown in Figure $2 \mathrm{~B}$, where the EDXS intensity of $\mathrm{Ti}$ and $\mathrm{Pb}$ is highest in the region labeled $\mathrm{X}$. The composition of this region is tentatively identified as PZT with $\mathrm{Pb}, \mathrm{Zr}$, and Ti elements also present in the region. However, the EDXS intensity for $\mathrm{Zr}$ is lower in the region labeled $\mathrm{X}$ when compared to adjacent regions. This could be due to the fact that the EDXS-SEM technique is based on the X-rays generated from bulk samples and therefore not sensitive to films less than $100 \mathrm{~nm}$ in thickness. [5] Also, it is known that the EDXS energy of Zr is very close to the energy for Ir. Electron energy loss spectroscopy (EELS) and EDXS in a transmission electron microscope (TEM) provide quantitative methods to identify the local composition of thin films. Thus, 
TEM-EELS and TEM-EDXS analysis are scheduled to be performed in order to positively identify the composition of the regions in Figure 1B.
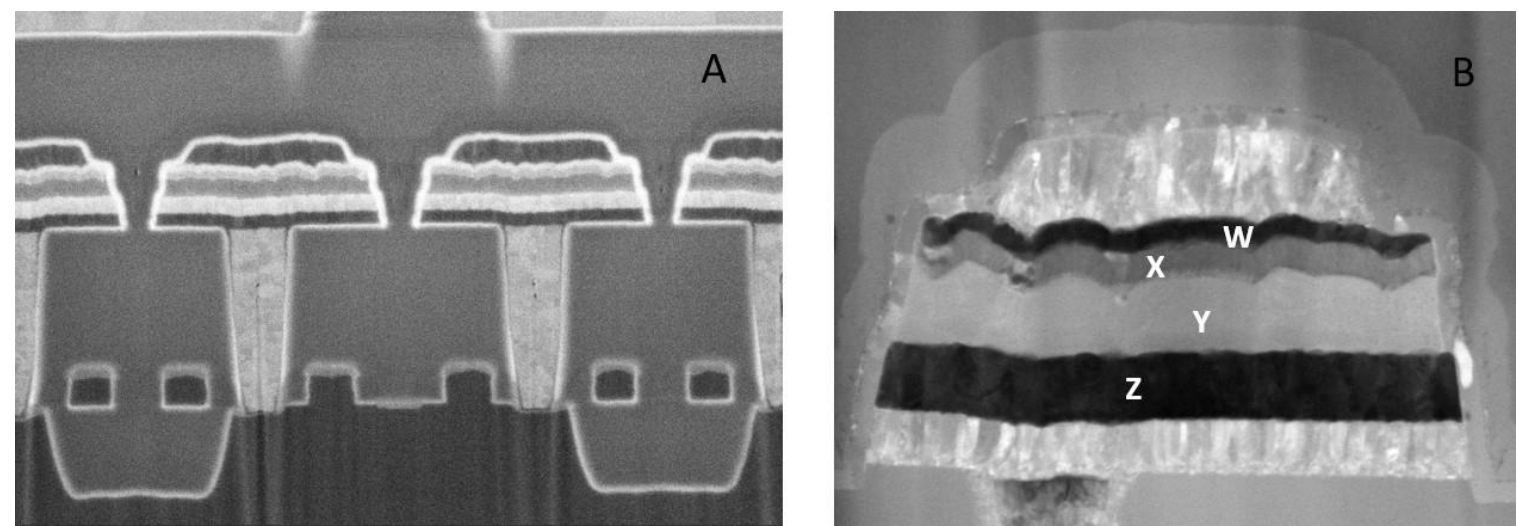

Figure 1. A) Cross-sectional SEM image demonstrating the FRAM capacitor sandwiched between the contact and the metal one layer. B) STEM image of the FRAM module depicting the polycrystalline and porous nature of various section of the FRAM capacitor.
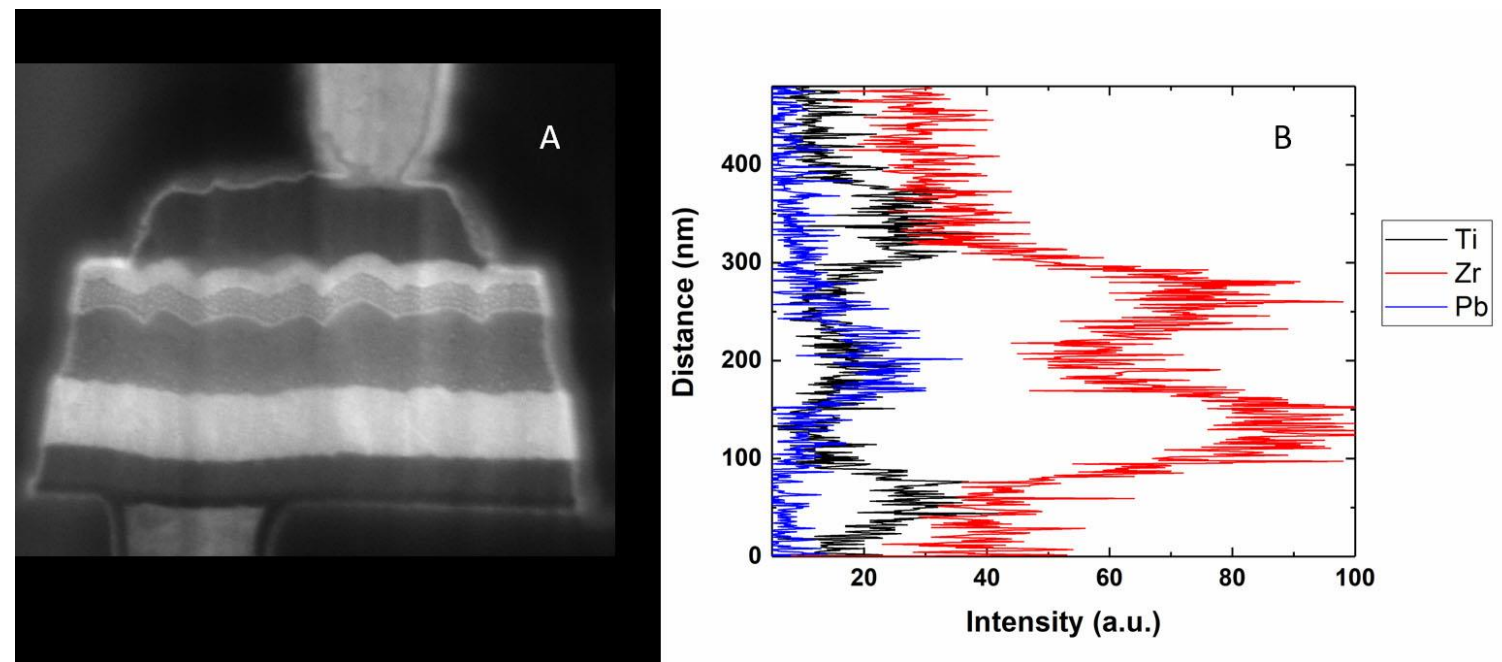

Figure 2. A) SEM image of FRAM part with a marked red line as a survey image during EDXS acquisition. B) Integrated EDXS profile taken from the labeled area in the SEM image.

\section{References}

[1] R. Whatmore (2017) Ferroelectric Materials. In: Kasap S., Capper P. (eds) Springer Handbook of Electronic and Photonic Materials. Springer Handbooks. Springer, Cham

[2] C. Yeh, et al. AIP Advances, 6, 035128, 2016.

[3] J. R. Devaney, et al. Failure Analysis Mechanisms, Techniques, \& Photo Atlas. 1983.

[4] D. C. Yoo, et al. Symp. On VLSI Tech. Dig., pp. 100-101, 2005.

[5] L. C. Yung, RSM 2013 IEEE Regional Symposium on Micro and Nanoelectronics, Langkawi, 2013, pp. 375-378. 